\title{
Medical photogrammetric measurement: overview and prospects
}

\author{
H.L. Mitchell ${ }^{\mathrm{a}, *}$, I. Newton ${ }^{\mathrm{b}}$
}

${ }^{a}$ School of Engineering, University of Newcastle, Newcastle, NSW 2308, Australia

${ }^{\mathrm{b}}$ formerly at Department of Geomatics, University of Newcastle upon Tyne, Newcastle upon Tyne, NE1 7RU, United Kingdom

\begin{abstract}
:
Since the earliest days of photogrammetry, there have been photogrammetrists who have directed research effort towards medical measurement. Although the specific motivation for these studies has not always been disclosed, it is probably because of the various benefits that photogrammetry can offer to humanity as a painless and non-invasive means of providing medical practitioners with spatial measurement relating to the human body. The intention of this paper is to reflect on the place of the many medical developments within the photogrammetric world. The various photogrammetric applications in medicine are summarised to identify the characteristics of medical photogrammetry, and it becomes evident that medical photogrammetrists have developed a range of body measurements using widely varying photogrammetric techniques, in response to the demands for specialised spatial measurement tools for a wide variety of medical ends. This volume of research activity has made medical measurement a substantial and varied sector of non-mapping photogrammetry. It is also clear that there are numerous challenges to the design of medical photogrammetric systems. They must give paramount consideration to the human patients and their comfort;
\end{abstract}

\footnotetext{
*Corresponding author: H.L. Mitchell, email: harvey.mitchell@newcastle.edu.au,
} 
they must yield not simply spatial data about the body but rather specific medical information. At the same time, it is of concern that non-photogrammetric scientists and engineers have developed medical measurement systems based on both photogrammetric and other optical techniques. Fortunately, photogrammetrists can also contribute to medical problems through their spatial data experiences. It is concluded that the many distinctive challenges mean that medical photogrammetry has not yet been as successful at changing the world as the extensive efforts applied to it suggest, but nevertheless medical photogrammetry deserves to be recognised as a category of close range photogrammetry which remains a valuable pursuit which should not be abandoned because of any difficulties.

Keywords: Medicine; photogrammetry;

Tel: +61-2-49216055; Fax: +61-2-49216991 


\section{Introduction}

Over the last century or more, the employment of photogrammetric measurement as a tool in disease diagnosis, patient treatment, motion analysis and medical research has appealed to a number of practical and theoretical photogrammetrists, as well as to medical instrumentation engineers and scientists. Reports of their activities, found in the medical and photogrammetric literature, show that the work has involved numerous parts of the human body, has been concerned a large variety of medical problems, and has been undertaken with a diverse range of photogrammetric approaches. A number of descriptions of medical photogrammetry exist as chapters in books on close range photogrammetry, for those wishing to learn about the techniques which have been used, (e.g. Newton, 1980; Newton \& Mitchell, 1996; Sheffer \& Herron, 1989). However, the aim of this paper is not to provide instructional photogrammetric tutoring or a detailed historical record of medical photogrammetric studies. It is to consider the position of medical photogrammetry within the photogrammetric world and to some extent also within the wider field of general medical measurement. In particular, the characteristics which distinguish it from other branches of photogrammetric measurement or which give it commonality with them are highlighted. The paper is intended to illustrate the diverse range of applications of medical photogrammetry, to help assess the place of medical photogrammetry within the broader photogrammetric arena, and, in this way, to provoke thoughts on the current status and future prospects for this application area. 


\section{An outline of medical applications of photogrammetry}

It is useful to precede reflections on the place of medical photogrammetry with a brief outline of some past medical photogrammetry projects, not to record the many applications but to provide some background to help extract the characteristics of the photogrammetric attempts to contribute to medical studies. A gauge of especially useful and effective photogrammetric research and development is whether it has been implemented for routine medical measurement, particularly if it is also used outside the developers' own institutions. Any medical measurement system that results in commercial implementation or production is seen as very successful. The latest interests in medical photogrammetry do not appear to be significantly different from those shown here.

For each application, the important matters to be considered are the impetus for the photogrammetric work, the challenges, and the alternative measurement tools. These, together with the highlights and the greatest successes, should help to explain why developments have occurred in the way that they have.

Providing a long bibliography would be easy. However the list of cited studies has been limited, preference being given to those that are the most instructive. Measurements of the human body which have not been carried out for medical purposes, but for art, forensic sciences or various other recording applications which have not been carried out with specific medical applications in mind have not been cited.

\subsection{The Face:}

Photogrammetric measurement has been used on the face more than on any other part of the body. Measurements have been made to monitor facial shape it changes over an extended period of time, through growth (e.g. Burke \& Beard, 1979) and during the treatment of 
various conditions (e.g. Gabel \& Kakoschke, 1996). Photogrammetry has also been used to investigate changes over short periods of time, such as before and after surgery, as surgical intervention to improve cosmetics or dis-functional jaw structures is quite common (e.g. Coombes et al., 1990). A report on a less common usage of facial measurement, to identify physical or psychology conditions within the population (Shaner et al., 2000) is informative, as it illustrates that some measurement applications are not always the most obvious ones.

Shaner et al. (1998a, 1998b) compare various direct and remote sensing methods of facial measurement, and it is apparent that there are few alternatives to optical methods for measuring the full face without distorting it or causing discomfort to the patient. The advantage of photogrammetry as a means of recording the full extent and detail of the face was seen well before digital technology emerged, (e.g., Burke \& Beard, 1967). However, providing the accurate and high-resolution spatial data which can depict the face with the very fine detail which is relevant to human recognition and cosmetics can be demanding, especially given its complex shape and the occlusions which can occur in stereophotographs. To be acceptable for use within medical organisations, measurement systems may need to supply facial features draped on a 3D model. Further, because facial measurement can be useful in surgical planning to provide both surgeons and patients with predictions of the outward changes expected from their operations, dynamic computer models may sometimes need to be an element of the measurement system.

It becomes apparent that any system satisfying all the requirements for facial measurement will be purpose-built, and not based on existing close range photogrammetric systems. Perhaps because of these various difficulties, and despite the demand and extensive development, photogrammetric methods are still not in widespread use. No commercial photogrammetric systems are known, but body measurement systems using scanning 
techniques are advertised, not only for medical purposes (see Cyberware, 2001, Apparel Research Network, 2002).

\subsection{The Back:}

The dominating need for back measurement is caused by the widespread occurrence of scoliosis, or curvature of the spine which often becomes apparent in teenage years, with both cosmetic and functional consequences. Measurements are needed to detect the condition and for monitoring the progress of treatment. Despite the apparent benefits offered by photogrammetry, the accuracy, and even the capability, of determining from the external shape measurement reliable values for spinal shape parameters for use in the disease's diagnosis and treatment is a matter of some argument. Even so, external measurement is preferred by many orthopaedic specialists because of the danger of excessive X-ray dosages with internal measurement systems, and photogrammetry can clearly compete with the cruder mechanical measurement tools that are used when radiography is to be avoided.

The quantity of surface shape studies is evidenced by the extensive mention of external back shape measurement in the proceedings of the long series of international meetings to consider scoliosis and related diseases (see International Research Society for Spinal Deformities, 2001). The moderate surface undulations of the back make it an ideal surface for photogrammetric recording, and even fully automated photogrammetry. Accordingly, many medical photogrammetric developments have related to the back, most using cast texture to provide detail suited to point matching. The benefit of measurement is indicated by the existence of a number of integrated measurement and analysis systems offered commercially. However, as for the face, photogrammetric systems are rare (but see e.g. ADAM Technology, 2001), and they must compete with other optical systems (see Bioengineering Technology and Systems, 2001). 


\subsection{Motion Studies:}

Interests in various gait problems, arising primarily from deformities or injuries, dominate human motion studies, while irregularities in walking patterns can also be relevant to other medical conditions, such as diabetes where the loss of blood circulation in the extremities can contribute to difficulty in feeling feet positions. The work of Holmes in 1863, who used stereopairs of photographs to study gait, is often quoted as the earliest application of photogrammetry in medical measurement, and his measurements led to an improvement in the design of artificial limbs for amputees maimed during the American Civil War. The possibility for producing high-speed imaging sequences is the obvious appeal of photogrammetry in this work. However, the photogrammetric processing is quite complex, because of the need for sophisticated target detection from sequences of images typically from multiple cameras and with images suffering occlusions in sequential images. Collections of useful papers on the subject are found in some conference proceedings (e.g. Walton, 1990). Despite the challenges, this application area has seen a more extensive implementation than any other has, and commercial systems for studies of walking or running are utilised widely in medical institutions around the world. Bioengineering Technology and Systems (2001), Qualisys AB (2001) and Vicon Motion Systems (2001) give details of systems which are available commercially.

Another group of movement studies by photogrammetrists relates to people involved in sport, notably athletics but also in golf, tennis and football, (Chikatsu et al., 1992; Chikatsu \& Murai, 1994), but these uses do not seem to have reached commercial levels. There have also been some less common movement studies, such as that described in Adams and Klein (1986) which provided measurements of the breathing cycle of babies, where again the crucial feature was the ability of the imaging methodology to avoid contact with the patient. These studies indicate that the extent of the demand for measurement is not always obvious. 


\subsection{Skin}

Sores, ulcers, wounds, melanomas and other skin conditions have been studied by photogrammetry, typically to monitor change with time. For this reason, photogrammetry's main advantage lies in the provision of an accurate record, but a significant challenge lies in providing cheap and simple techniques for use in clinics outside hospitals. The only alternative seems to be simple observation, sometimes accompanied by photography, but despite a widespread interest in this work, (e.g. Eriksson et al., 1979; Boersma et al., 2000), photogrammetric systems are not in common use.

\subsection{Teeth}

Measurements of teeth are utilised to detect the occurrence of wear, erosion and abrasion in both natural tooth surfaces and in tooth restoration materials, but the repetitive measurement has generally been carried out on teeth replicas derived from castings, rather than on teeth in situ. Needless to say, the photogrammetry has typically used microscope imagery and has been pursued because of the difficulty of finding alternatives that can be used with such small objects. The common difficulties faced by photogrammetric solutions here are the need to determine camera orientation parameters and a lack of texture on the objects. Chadwick (1992) has provided a review of the use of photogrammetry for such purposes. As surveys such as that by Azzopardi et al. (2000) show, photogrammetry faces competition from a variety of sophisticated optical and mechanical devices in such investigations.

\subsection{Other Measurements of the Body:}

Photogrammetry has been applied to many other parts of the body but without the emphasis that has been given to the topics mentioned above. Interesting applications have involved eyes 
(Takamoto \& Schwartz, 1986, Banda \& Muller, 1990), feet, breasts, abdomens (Karras \& Petsa, 1992) and assorted sites for which prostheses are to be designed. Photogrammetry offers various advantages, centred on quick non-contact recording, but no photogrammetric systems are known to be in widespread use yet.

Positioning of the body (e.g. Baroni et al., 2000) seems to be a most useful application of photogrammetry as it is difficult to achieve by alternative methods.

\subsection{Measurements within the Body}

The benefits offered by imaging give photogrammetry an application in recording surgical processes, sometimes for teaching purposes. Some fully digital photogrammetric methods (e.g., Adams et al., 1994; Tredwell et al., 1995) have been developed for surgical use within specific hospitals. Multiple imaging systems are used by Valstar et al., (2002b), who also refer to examples of camera-based systems for computer-assisted surgery which are available commercially.

\subsection{X-Ray methods}

The use of radiographs to find three-dimensional information can be of interest because of the use of photogrammetric reduction principles and practices. The extent of use in medical institutions is not clear, but numerous publications exist in the medical and photogrammetric literature, often referring to the back (e.g. Merolli et al., 1995; Lofgren et al., 2000), the face (e.g. Thomas et al., 1996) and to studies of movements of implants (e.g. Valstar et al, 2002a). It is of photogrammetric interest that in a number of the facial cases, radiography has been used in conjunction with external photography. 


\section{Special Characteristics of Medical Photogrammetry}

It becomes apparent that medical photogrammetry has certain characteristics, which distinguish it from other photogrammetric applications, such as those in mapping, architecture, or industry. These characteristics can be used to assess the place of medical photogrammetry within the wider photogrammetric world.

1) Medical photogrammetry has a wide scope. Although photogrammetric measurement is particularly relevant to orthopaedics and anatomy, it can nevertheless make contributions to ophthalmology, neurology, dentistry, occupational therapy, and other studies related to human health, such as ergonomics. It can assist in the detection, diagnosis and treatment of medical conditions, in epidemiological studies and in medical research. Medical photogrammetry is particularly useful with those medical syndromes that manifest themselves in visible outcomes, yet it also finds application with medical conditions that are not immediately perceptible. For example, measurements of the face can be useful in psychological studies and gait measurement in studies of diabetes. Photogrammetry is not even limited to external measurement, as its uses in surgery indicate. Measurements may be carried out it on both sick and healthy people; they may involve a variety of persons from medical community, including medical practitioners or paramedical professionals; and they may be carried out inside or outside large and small hospitals, clinics and other medical institutions. Medical photogrammetry can even extend into sports medicine, dentistry and, if necessary, veterinary science. The scope of medical photogrammetry is, therefore, much wider than may at first be anticipated

2) The consideration for human patients is crucial: Photogrammetry's primary advantage in medical applications arises from its basis in imaging, more often than with aspects of the 
subsequent processing of the images. As an imaging technique, it not only offers short patient involvement times, but it also avoids contact with the patient and thereby avoids the risk of deforming the area of interest and spreading infection. Moreover, image acquisition is generally a simple process, using small and familiar equipment that is not daunting to patients. The general lack of intrusion largely avoids any physical and mental discomfort to patients. In motion studies, the lack of contact avoids the use of instruments that can interfere with the motion under study. While other photogrammetric applications utilise the speed and remoteness of imaging systems, consideration of the nature of the equipment itself is rarely of such significance as it is in medical work. Medical photogrammetry is unique in that it deals with human patients and it is necessary to be concerned about their convenience, comfort, dignity, safety and health.

3) It is necessary for the measurement system to provide medical output, in addition to the spatial data: Medical information is often not provided by the raw spatial data, so a common requirement of medical applications is that medically relevant data be extracted from it. This can often create the most difficult part of any application. For example, while the human back is seen as an ideal surface for close range photogrammetric recording, inferring three-dimensional spinal shape from back shape measurements is not so easy; a measured tooth shape does not provide any immediate information about its erosion qualities; and gait observations on a moving person, difficult enough in themselves, do not offer direct information about the medical condition that causes the movement problem. Even the presentation of any basic spatial information requires considerations of presentation to those not normally familiar with it, as recognised by Chadwick \& Mitchell (1999). So, while an architectural survey may immediately provide visual information of value, and a map is of immediate use to a walker, virtually all medical photogrammetric 
measurements require further interpretation and analysis to allow meaningful information to be given to the end-user. Only if this additional information is provided and understood will the photogrammetry be utilised in practice. The interested photogrammetrist must therefore recognise the need to incorporate this additional burden in developing any medical measurement system.

4) The photogrammetrist is not the sole party in the development: The arguments given in (3) make it clear that developments in medical photogrammetry invariably require both photogrammetric and medical personnel to be involved to provide a system which is approved by all parties. The users of the results may be involved in health in various ways, as physicians, surgeons, clinicians, medical researchers, bio-mechanical engineers, occupational therapists, and so on. It is obviously not practicable for photogrammetrists to accurately perceive all the requirements of the patient and medical practitioner. Any medical photogrammetric development therefore involves various parties who must clearly communicate to establish the requirements, limitations and opportunities of the envisaged measurement system. Failure to communicate can lead to missed opportunities and wasted effort, and photogrammetrists need to be more proactive here.

5) Medical instrumentation scientists and engineers have developed medical photogrammetric systems: The difficulties caused by the arguments given in (3) and (4) mean that it can be easier for scientists and engineers working in the medical instrumentation field to develop medical photogrammetric systems than for photogrammetrists to do so. More specifically, research and development groups with a background in medical physics, computer engineering and so on, have successfully developed and implemented more photogrammetric systems (especially commercially 
successful systems) than have photogrammetric scientists (see Bioengineering Technology and Systems, 2001, and Vicon Motion Systems, 2001). A literature survey of medical photogrammetry is not complete without a search of medical and medical instrumentation literature, an exercise which can bring photogrammetrists into contact with sources which were previously unfamiliar. The fact that photogrammetrists have not been exclusively successful in developing measurement systems in this field is a cause for reflection about the role of photogrammetry within medicine.

6) Spatial measurement in medicine lacks alternative tools: It is ironic that the field of medicine, which is typified by expensive, high-technology equipment, should offer development opportunities to methodology based on imaging and optical techniques, precisely because of the lack of measurement alternatives. For the architectural or industrial surveyor or the mapmaker, alternative surveying tools, whether theodolites, EDM, or GPS, offer a means of carrying out measurements if photogrammetry is not available. But in medicine, it is often found that alternatives are relatively crude, typically being simple geometrical devices, including callipers, tapes, protractors and sometimes nothing more than un-quantified observation by the human eye. Newton \& Mitchell (1996) attribute this state of affairs to the fact that the spatial measurement is generally not crucial to saving lives. This latter point has probably had more influence on the development of medical photogrammetry than the apparent opportunity offered by photogrammetry to medicine to replace simple tools.

7) Medical measurement needs specialised photogrammetric systems: Photogrammetric systems for mapping or non-medical close range applications are rarely found to be suited to immediate application in medicine. A reason for this is that medical data must be 
provided, but the main reason is that medical systems have to be made usable within hospitals and other institutions, by medical staff rather than by photogrammetrists.

8) The photogrammetric techniques vary widely: The techniques developed for medical purposes may involve measurement of surfaces or targetted points. Surface measurements typically involve added texture but in some cases such as skin lesions, they may not. Objects may be as small as teeth or as large as the entire person. Photogrammetric variety ranges from systems involving high-speed measurement of targeted objects to surface measurement with structured-light assistance. Sometimes the benefit arises from the speed of motion imaging. In some cases, such as facial measurement, benefits lie in the provision accuracy and detail. Generally, however, accuracy requirements are low compared with those expected when mapping from aerial photographs. For other applications, the medical practitioner can gain advantages through the automation of digital photogrammetry which takes away the risk of judgement and provides a better cost/benefit ratio.

9) Photogrammetry faces competition from allied optical methods: In many of the medical measurement problems which involve surface measurement (ie. excluding the applications which involve targets, surgical recording and so on), the benefits of photogrammetry's quick, non-contact recording are also offered by a number of other non-photogrammetric techniques. They are frequently based on structured light methods (especially for back shapes, e.g. Hierholzer, 1989) or laser scanning (for faces and backs, see Cyberware, 2001), and these methods have even been investigated for the positioning of teeth within the mouth (Commer et al., 2000). Uses are also found for moiré fringing (notably for back measurement, see Suzuki et al., 1995), despite the method's inability to provide 
unambiguous quantitative data. In fact, it is apparent that most of the successful commercial surface measuring devices offered to the medical community are based on optical and imaging techniques which avoid photogrammetry's need for point recognition and matching. However, experimenters with a photogrammetric background can easily adapt to other optical techniques such as these, because of the common concepts of intersecting rays, and common imaging hardware and image processing software. Even the problems of occlusions, presentation and extraction of medical information are shared. Unfortunately, it cannot be assumed that photogrammetric systems will be welcomed without qualification by the medical profession.

10) Photogrammetrists can contribute without necessarily taking photogrammetric measurements: Some photogrammetrists' contributions to medical studies have been through their experiences in spatial data handling rather than by assistance with measurement processes. Examples include the role of Adams (Adams et al., 1994) in the development of a cerebral probe positioning; Karras and Petsa (1992) used photogrammetry but their primary contribution to studies of pregnant women arguably lay in the use of surface registration; Mitchell has used surface matching in tooth erosion studies which have involved mechanical and not photogrammetric measurement developed by medical colleagues, (Mitchell \& Chadwick, 2000).

\section{Conclusions}

The characteristics of medical photogrammetry as outlined above can help us to identify its place within the wider world of photogrammetry. It is concluded that medical photogrammetry constitutes a distinctive category within close range photogrammetry, 
because of the unique challenges which it faces. Challenges are created by the need to work so closely with an unfamiliar profession and industry. They also arise from the requirement to provide equipment which is suited to the measurement of sensitive human beings within medical environments. Further difficulties are caused by the impracticality of using existing commercial photogrammetric systems, and by the constrains of providing low-cost systems. At the same time, photogrammetry faces competition from alternative optical techniques, with extensive numbers of non-photogrammetrists successfully developing medical measuring equipment. Because of these obstacle to their implementation in real and commercial situations, only a small proportion of the medical photogrammetric research and development has been implemented for genuine medical measurement. It can even be argued that medical photogrammetry has not yet been as successful at changing the world as it deserves, given the extensive efforts applied to it. The difficulties of ensuring successful development have been recognised earlier, and have led to comments on the suitability of various medical problems to photogrammetric development by Mitchell \& Leemann (1996), who examined the variety of medical measurement applications via a questionnaire, in search for the medical applications which most deserved photogrammetrists' time. Their conclusion was that photogrammetrists should be wary of attractive projects which are unlikely to lead to successful implementation. Photogrammetrists are therefore advised to focus their developments in areas that will see genuine medical usage, to avoid wasting their efforts.

Despite the obstacles, the extensive medical budget of many societies should encourage this type of photogrammetry. It should retain its attraction into the future, especially as the benefits of digital methods and automation grow, and opportunities for useful and interesting developments in medical measurement remain. Better communication between photogrammetrists and the medical community would help and attendance at bio-medical conferences and the submission of papers to bio-medical journals can assist in this direction. 
Undoubtedly, medical photogrammetry appeals to some photogrammetrists and nonphotogrammetrists because of its apparent benefit to society, and while external measurements are rarely involved in saving lives, it is nevertheless important that medical photogrammetry be pursued because of these benefits.

\section{References}

ADAM Technology, 2001. http://www.adamtech.com.au/3dm/back.html, accessed 1 August 2001.

Adams, L.P. \& Klein, M., 1986. Biostereometric methods for the study of body surface motions during breathing. International Archives of Photogrammetry \& Remote Sensing 26 (5), 263-270.

Adams, L.P., van Geems, B.A., Jaros, G.G., Peter, J. \& Wynchank, S., 1994. A stereometric controlled pointing device for surgical use. International Archives of Photogrammetry \& Remote Sensing 30(5), 1-8.

Apparel Research Network, 2002. http://arn.iitri.org/docs/scan/links/index.html, accessed 11 February 2002.

Azzopardi, A., Bartlett D.W., Watson T.F., Smith, B.G., 2000. A literature review of the techniques to measure tooth wear and erosion. Eur. J. Prosthodont. Restor. Dent. 8(3), 9397.

Banda, F.A.S. \& Muller, J-P., 1990. Cornea shape measurement. International Archives of Photogrammetry \& Remote Sensing 28(5/1), 720-726.

Baroni G., Ferrigno G., Orecchia R., Pedotti A., 2000. Real-time three-dimensional motion analysis for patient positioning verification. Radiotherapy \& Oncology 54(1):21-27 
Bioengineering Technology and Systems, 2001. http://www.bts.it/bts/products.htm, accessed 1 August 2001.

Boersma, S. M., van den Heuvel, F.A., Cohen, A.F. \& Scholtens, R.E.M., 2000. Photogrammetric wound measurement with a three-camera vision system. Int. Archives Photogrammetry \& Remote Sensing 33(5A):84-91.

Burke, P.H. \& Beard, L.F.H., 1967. Stereophotogrammetry of the face. American Journal of Orthodontics 53(7), 769-782

Burke, P.H. \& Beard, L.F.H., 1979. Growth of soft tissues of the face in adolescence. British Dental Journal 146(8), 239-246.

Chadwick, R.G., 1992. Close range photogrammetry - a clinical dental research tool. Journal of Dentistry 20, 235-239.

Chadwick, R.G. \& Mitchell, H.L., 1999. Presentation of quantitative tooth wear data to clinicians. Quintessence International 30, 393-398.

Chikatsu, H. \& Murai, S., 1994. Application of image analysis to rowing dynamics using video camera. International Archives of Photogrammetry \& Remote Sensing 30(5), 35-40.

Chikatsu, H., Turuoka, M \& Murai, S. 1992. Sports dynamics of Carl Lewis through 100m race using video imagery. International Archives of Photogrammetry \& Remote Sensing 29(B5), 875-879.

Commer, P., Bourauel, C., Maier, K. \& Jäger, A., 2000. Construction and testing of a computer-based intraoral laser scanner for determining tooth positions. Medical Engineering \& Physics 22, 625-635.

Coombes, A.M., Linney, A.D., Richards, R. \& Moss, J.P., 1990. A method for the analysis of the 3D shape of the face and changes in the shape brought about by facial surgery. In: Herron, R.E. (Ed.), Biostereometric Technology \& Applications. SPIE 1380, 180-189. Cyberware, 2001. http://www/cyberware.com/products/index.htm, accessed 1 July 2001. 
Eriksson,G., Eklund, A.-E., Torlegard, K. \& Dauphin, E., 1979. Evaluation of leg ulcer treatment with stereophotogrammetry. British Journal of Dermatology 101(2), 123-131.

Gabel, H. \& Kakoschke, D., 1996. Photogrammetric quantification of changes of soft tissue after skeletal treatment of the facial part of the skull. International Archives of Photogrammetry \& Remote Sensing 31(B5), 188-193.

Hierholzer, E., 1989. Raster photogrammetry: systems and applications. In: Karara, H.M. (Ed.), Non Topographic Photogrammetry, American Society of Photogrammetry \& Remote Sensing, Falls Church, pp. 265-278.

International Research Society for Spinal Deformities, 2001. http://www.liv.ac.uk/HumanAnatomy/phd/irssd/pastmeet.html, accessed 1 August 2001.

Karras, G.E. \& Petsa, E., 1992. On detection of deformation in reconstructed body models. Proc. Int. Symposium on 3-D Scoliotic Deformities, 28-30 June, Montreal, Canada, Danserau, J. (Ed.), Gustav Fischer Verlag, pp. 370-375.

Lofgren H., Johannsson, V., Olsson T., Ryd, L., Levander, B., 2000. Rigid fusion after cloward operation for cervical disc disease using autograft, allograft, or xenograft - A randomized study with radiostereometric and clinical follow-up assessment. Spine, 25(15):1908-1916.

Merolli, A., Tranquilli Leali, P. \& Aulisa, L., 1995. Considerations on the clinical relevance of photogrammetric error in 3-D reconstruction of the spine based on routine X-ray films. In: d'Amico, M., Merolli, A. \& Santambrogio, G.C. (Eds.), Three Dimensional Analysis of Spinal Deformities. IOS Press, Amsterdam, pp. 185-190.

Mitchell, H.L. and R.G. Chadwick, 2000. Digital photogrammetric concepts applied to surface deformation studies Geomatica. 53(4), 405-414

Mitchell, H.L. \& Leemann, T., 1996. Issues in medical photogrammetry in the digital imaging era. International Archives of Photogrammetry \& Remote Sensing 31(B5), 362-367. 
Newton, I., 1980. Medical photogrammetry. In: Atkinson, K.B. (Ed.), Developments in Close Range Photogrammetry - I. Applied Science Publishers, London, pp. 117-148.

Newton, I. \& Mitchell, H.L., 1996. Medical Photogrammetry. In: K.B. Atkinson (Ed.), Close Range Photogrammetry and Machine Vision. Whittles Publishing, Latheronwheel, pp. 303327.

Qualisys AB, 2001. http://www.qualisys.se, accessed 1 August 2001.

Shaner, D. J., Peterson, A. E., Beattie, O. B. \& Bamforth, J. S., 1998a. Facial measurements in clinical genetics: How important are the instruments we use? American Journal of Medical Genetics 77, 384-390.

Shaner, D. J., Bamforth, J. S., Peterson, A. E. \&Beattie, O. B., 1998b. A comparison of photogrammetric and caliper-derived measurements. American Journal of Physical Anthropology 106, 547 - 552.

Shaner, D. J., Peterson, A. E., Beattie, O. B. \& Bamforth, J. S., 2000. Assessment of soft tissue facial asymmetry in medically normal and syndrome-affected individuals by analysis of landmarks and measurements. American Journal of Medical Genetics 93, 143-154.

Sheffer, D.B. \& Herron, R.E., 1989. Biostereometrics. In: Karara, H.M. (Ed.), NonTopographic Photogrammetry. American Society of Photogrammetry and Remote Sensing, Falls Church, pp. 359-366.

Suzuki, N., Ono, T., Tezuka, M. \& Kamiishi, S., 1995. Moire topography and back shape analysis. In: d'Amico, M. , Merolli, A. \& Santambrogio, G.C. (Eds), Three Dimensional Analysis of Spinal Deformities. IOS Press, Amsterdam, pp. 124-130.

Takamoto, T. \& Schwartz, B., 1986. Three-dimensional mapping of retinal vessels in ophthalmology. International Archives of Photogrammetry \& Remote Sensing 26(5), 349354. 
Thomas, P.R, Newton, I \& Fanibunda, K. 1996. Evaluation of a low cost digital photogrammetric system for medical applications. International Archives of Photogrammetry \& Remote Sensing 31(B5): 405-410.

Tredwell, S.J., Booth, K.S., Reilly, C., Sawatzky, B.J. \& Jang, S, 1995. Three dimensional analysis of scoliosis surgery using stereophotogrammetry. In: d'Amico, M. , Merolli, A. \& Santambrogio, G.C. (Eds), Three Dimensional Analysis of Spinal Deformities. IOS Press, Amsterdam, pp. 95-100.

Valstar, E.R., Botha, C.P., van der Glas, M., Rozing, P.M., van der Helm, F.C.T, Post, F.H., Vossepoel, A.M., 2002a. Towards computer assisted surgery in shoulder joint replacement. ISPRS J. Photogrammetry \& Remote Sensing, in press.

Valstar, E.R., Botha, C.P., van der Glas, M., Rozing, P.M., van der Helm, F.C.T, Post, F.H. \& Vossepoel, A.M., 2002b. Towards computer assisted surgery in shoulder joint replacement. ISPRS J. Photogrammetry \& Remote Sensing, in press.

Vicon Motion Systems, 2001. http://www.vicon.com, accessed 1 July 2001.

Walton, J.S. (Ed.), 1990. Image-based motion measurement, SPIE 1356, San Diego. 144 pages. 